during the two days prior to the symposium, and the Sub-Committees on Cartographic Problems in the Arid Zones, and on the Socio-Economic Problems of the Arid Zones, also held meetings at this time.

During the symposium there was an exhibition of maps, photographs and publications illustrating the activities of Unesco, the member States and other bodies; two excellent films were also shown-the Unesco film, "Arid Lands", produced by World Wide Pictures, Ltd., based mainly on scenes and research activities in Pakistan, Israel and Morocco, and a Russian film depicting arid zone development, in respect of irrigation, dry farming and stock raising, in the Karakum and other deserts of the U.S.S.R. Also, a party of delegates visited the phytotron being set up at Gif-sur-Yvette by the Centre National de la Recherche Scientifique; the studies in plant physiology at the phytotron, with special reference to growth under varying conditions of light and temperature, were demonstrated by the director, Prof. P. Chouard.

In respect of the high standard of the papers presented, of the many countries of the arid zone represented, and of the free discussions that followed the papers, the symposium marked an important stage in the study of the arid zones; moreover, it afforded a valuable review of present knowledge and of the scope and nature of future research, and it clearly achieved its objective in presenting, for the consideration of the governing bodies of Unesco, a unanimous and broadly based opinion on the importance of maintaining arid zone research and training at a high level.

Whatever may be the final decision, Unesco's achievements to date will rank high as a contribution to science in making known the problems of the arid zones, and in assisting in their scientific investigation, thereby leading also to the increased economic and social development of these lands.

\section{F. Dixey}

\title{
SELF-REGULATION IN LIVING SYSTEMS
}

T THE Ottawa symposia on "Self-regulation in Living Systems" organized by Dr. D. K. C. MacDonald of the National Research Council shows signs of becoming an annual series (Nature, 184, $239 ; 1959$, and 183,$370 ; 1959)$. The third meeting was held on March 4, in Montreal, with the Montreal Neurological Institute and McGill University as joint hosts. The attendance exceeded thirty people, representing most medical branches, physies and engineering. The theme was "Tremor", and on this occasion the topics of discussion were introduced by clinicians and illustrated with the willing co-operation of patients. Discussion during the day was general and spirited.

Dr. F. L. MacNaughton (Montreal Neurological Institute) introduced the subject of 'clonus' (alternating contractions and relaxations of muscle). A patient with ankle clonus was introduced and subsequent discussion on the mechanism of oscillation was therefore restricted to this example. The oscillation could be initiated by a sharp application of force to the ball of the foot, and was sustained as long as the force remained. Simultaneous but asynchronous oscillation of both feet could be produced. The oscillation frequency was about 4 c.p.s. and increased only slightly with pressure. The disease had been caused by a spinal tumour pressing on the spinal cord ; the tumour had recently been removed and there was some slight improvement in the patient's condition. She was paralysed from the waist down and could not feel the clonus directly. MacNaughton explained that the disease was known. to be caused at the spinal cord level, rather than higher in the central nervous system. Ankle clonus was produced mechanically by involuntary contractions of the leg's rear lower muscle (gastrocnemius) followed by relaxation under action of the external force. Clonus was not produced by a coupled oseillation between the rear and front leg muscles, and disappeared if the afferent stretch receptors in the gastrocnemius were cut. Any healthy person can produce clonus, for example, by trying to lift an unliftable weight.
After this discussion Dr. MacDonald (National Research Council) exhibited a simple closed-loop system as an illustration of the instability features discussed above. In this demonstration a galvanometer mirror deflected a light beam so as to follow the physical movement of a photo-cell. Normal stable following was produced by negative feedback at some gain. Sufficiently increased gain, however, drove the light spot into a tremor, as did increase of the controllable time-delay in the circuit.

Dr. Burns (McGill) discussed the physiology of the basic causal loop; that is, muscle-stretch receptormultiple afferent nerves - spinal cord-multiple motor neurons-muscle. With nerve propagation speed of order $10^{2} \mathrm{~m}$./sec. and nerve-length of order $\mathrm{I} \mathrm{m}$., the propagation time of the nerve in the loop is 20 msec. (2 nerves); other lag-times in the circuit are negligible. This loop delay-time would produce an oscillation of 25 c.p.s. if it were the direct cause of clonus, but this provides poor correlation with the observed 4-10 c.p.s., and of course this closed-loop action is the same in normal healthy voluntary actions.

The situation is complicated by the fact that the components of a multiple-nerve system do not fire simultaneously, but rather in a statistical pattern approaching a Gaussian distribution of frequency, and propagation velocities are also distributed about a mean in the range $70-120 \mathrm{~m}$. $/ \mathrm{sec}$. Furthermore, the excitability of a nerve follows a time pattern after firing as indicated in Fig. 1.

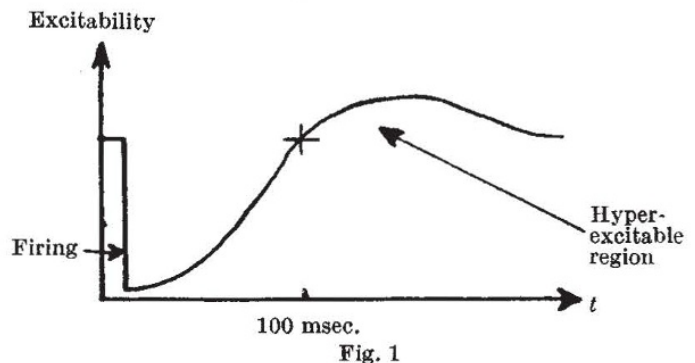

Fig. 1 
In discussion, Dr. Burns wondered whether the nerves in clonus might fire almost simultaneously due to hyperexcitability. The observed oscillation frequency corresponds well to a closed-loop oscillation based on the 100 -msec. time-delay to hyperexcitability in a nerve after firing.

In the afternoon, Dr. MacNaughton introduced a patient with Parkinson's discase. The particular symptom studied was a tremor of the thumb and forefinger, in the frequency range $3-5$ c.p.s. Amplitude increased with concentration, excitement and fatigue, but disappeared during sleep. Applica. tion of a heavy weight to the hand only reduced the amplitude. Purposeful action of the hand was quite possible and tremor decreased during a 'slewing' action, but increased, for example, as the hand approached the pen it was going to pick up.

Dr. Blundell (Montreal Neurological Institute) discussed the tremor problem from the point of view of the surgeon, particularly in relation to Parkinson's disease. Surgical possibilities are: (1) To cut the motor nerves; but then no movement is possible. (2) To cut the afferent nerves. This removal of feedback would be justified in extreme cases if it overcame incapacitating tremor. This is true of clonus, but in one known operation on a Parkinsonian case, tremor remained, although at a different amplitude. (3) To cut in the area of the central nervous system. This is the current field of research. Results so far are rather conflicting, and there is insufficient knowledge for confident surgery. Dr. Blundell then detailed the following possibilities as tremor producers: (1) scrvo-loop oscillations; (2) rhythmic oscillations, for example, by a steady driving mechanism in the brain; (3) hyperexcitability of cells, due to disease.

Dr. Burns also developed the physiology of muscle action somewhat further. Recently a second loop (the 'gamma' loop) has been identified by which 'small' motor neurons are fired from the spinal cord to contract the fibre which is measured by the length receptor. In addition, the afferent nervos from the stretch receptors communicate with the central nervous system. In turn the central nervous system communicates with both the 'big' and 'small' motor neurons. Several more loops are thus added, and much remains to be learned of the combined control action.

Dr. Jasper (University of Montreal) described extensive experimental measurements on tremor, being carried out at the University of Montreal, using micro-electrodes. Frequency measurements of the action potential of muscles in clonus and Parkinson's disease look exactly alike. On the other hand, the cortical phenomena seem different. Some 15-20 per cent of the cortex cells seem to show activity correlated with tremor, but not enough is yet known. In monkeys, tremor has been produced by a small cut in the brain in the appropriate area; a 'large' cut can then remove the tremor.

Finally, Dr. J. A. Tanner (National Rescarch Council) roported the work being done by Massachusetts Institute of Technology and Yale University on measuring the impedance of the wrist and finger extremitics when subjected to sinusoidal or stochastic vibrations. The impedance falls with frequency as might be expected for an inertial load, except that in healthy people there are resonant peaks in the 7 and 17 c.p.s. regions. These are as yet unexplained. In patients with tremor the major resonance is due to the tremor, but at a different frequency than the tremor itself, lying between the 7-17 c.p.s. peaks. This overshadows the other resonances, naturally enough.

A spirited discussion followed concerning the relative usefulness of the 'black box' technique as illustrated by the impedance measurements just described, and the classic analysis-techniques in which individual elements are isolated and their characteristics learned before they are combined together. Everyone agreed that the latter method is ideal if it can be used. Opinion was divided as to whether the 'black box' techniques could be useful, since the 'black box' may often be altogether too complex. I myself foel that both approaches are useful and must be pursued in parallel. This in fact is done of necessity in both engineering and medicine at least.

J. H. Mirsum

\section{SCHIZOPHRENIA AND LOW-GRADE MENTAL DEFICIENCY IN SWEDEN}

\begin{abstract}
A CLINICAL and genetico-statistical analysis of schizophrenia and low-grade mental deficiency of a large rural population of 25,000 people in Sweden indicated that altogether 1,790 individuals belonging to 231 families were suffering from schizophrenia, and 1,541 individuals belonging to 215 families were low-grade mental defectives*.

Of the cases of schizophrenia, 50 per cent were classified as hebephrenic, 33 per cent as catatonic, 10 per cent as paranoic and 7 per cent as atypical. 70 per cent of the cases had symptoms generally described as catatonic, but only 20 per cent had thern in connexion with the onset of the psychosis.

* A Clinical and Genetico-Statistical Study of Schizophrenia and Low-Grade Mental Deticiency in a Large Swedish Rural Population. By Bertil Hallgren and Torsten Sjögren. Acta Psychiatrica et Neuro(Copenhagen: Ejnar Munksgaard, 1959.)
\end{abstract}

The mean age at onset of schizophrenia among females, 32 years, is significantly higher than that, 28 years, among males. The psychosis had an acute onset in approximately 40 per cent of the cases and a periodical course in many cases, there being no sex difference. There is thus a positive relationship between acute onset of schizophrenia and periodical course of the psychosis.

There was a statistically significant association between schizophrenia and mental deficiency. Such an association has not been statistically established previously. The morbidity-rate for montal deficiency --including high-grade defectives-among the schizophrenic propositi is $10.5+1.9$ per cent as against a risk of 3 per cent estimated for the general population. The morbidity-rate for mental deficiency among the sibs of propositi was no higher than the 